\title{
Sonographic Grading of Renal Parenchymal Changes and its Comparision with Estimated Glomerular Filtration Rate (EGFR) Using Modified Diet in Renal Disease Formula
}

\author{
Prashanth K S ${ }^{1}$, Ravi ${ }^{2}$, Chandrashekar H M ${ }^{3}$ \\ ${ }^{1}$ Resident, Department of Radiology, ${ }^{2}$ Prfessor and Unit Head, Department of Radiology, ${ }^{3}$ Assistant Prfessor, Department of \\ Radiology, Bangalore Medical College and Research Institute, Bengaluru, Karnataka, India
}

Corresponding author: Dr. Prashanth KS, No. 306, BMCRI PG Mens Hostel, Near Royan Circle, Chamarajpet, Bangalore-560018, India

DOI: http://dx.doi.org/10.21276/ijcmsr.2020.5.2.4

How to cite this article: Prashanth K S, Ravi N, Chandrashekar H M. Sonographic grading of renal parenchymal changes and its comparision with estimated glomerular filtration rate (EGFR) using modified diet in renal disease formula. International Journal of Contemporary Medicine Surgery and Radiology. 2020;5(2):B13-B16.

\section{A B S T R A C T}

Introduction: The sonographic findings are of help in evaluating the nephrological diseases. Glomerular filtration rate is another parameter for assessing the reserved renal function and an indicator of prognosis. Study objectives were to evaluate the relevance of sonographic grading of renal parenchymal changes in assessing the severity of the renal disease and comparing it to the eGFR calculated using MDRD formula based on the age, gender and serum creatinine value of the patient.

Material and methods: The study was conducted in the Department of Radiology, in collaboration with Department of Medicine and Nephrology, Bangalore Medical College Hospital, Bangalore, India. The adult patients with suspected kidney disease referred from the department of medicine and nephrology for sonography of abdomen were the study participants. We conducted a cross-sectional study from November 2017 to May 2019 and data was collected for 80 patients during study period. The echo texture of spleen is used to compare the renal cortical echotexture. Then the serum creatinine, age, gender were documented for all study participants. eGFR is calculated using modified diet in renal disease (MDRD) formula which gives eGFR in $\mathrm{ml} / \mathrm{min} / 1.73 \mathrm{~m}^{2}$.

Results: A total of 80 patients were our study participants, out of which $47(67.1 \%)$ were males and $23(32.9 \%)$ were females. The mean age of the study participants was $55.45 \pm 8.35$ years (range: $40-72$ years). There was no specific pattern of sonographic grading in different age groups. The findings were not gender dependent and the findings were the same in both sexes. On sonographic evaluation of our study participants; $14(20 \%)$ cases showed grade " 0 ", $11(15.7 \%)$ cases showed grade "1", 30(42.9\%) cases showed grade "2" and15 (21.4\%) cases showed grade " 3 " renal parenchymal changes.

Conclusion: eGFR is an important factor which can be used as a factor to predict prognosis in renal diseases. Sonography is a good non-invasive diagnostic tool for evaluation of renal disease. There is good correlation between the sonographic grading and eGFR using MDRD formula. We conclude that by evaluating the kidneys with Sonography and calculating eGFR using MDRD formula the renal status will be more accurately interpreted.

Keywords: Sonographic Grading, Renal Parenchymal Changes, Estimated Glomerular Filtration Rate (EGFR), Diet, Renal Disease Formula

\section{INTRODUCTION}

Sonography is the imaging modality of choice in suspected cases of acute and chronic kidney diseases. The sonography has the advantage of affordability, availability, lack of ionizing radiation, portability with option of conducting investigation at patient's bedside and repeatability, for all these reasons sonography is the most useful investigating tool in evaluating kidney diseases. ${ }^{1}$ The sonographic findings of renal diseases are nonspecific, but these findings are of enormous help in evaluating the nephrological diseases. ${ }^{2}$ Sonography is commonly used to assess the kidneys and sonographic grading of renal parenchymal changes. Glomerular Filtration
Rate (GFR) is a calculation that determines how well the blood is filtered by the kidneys, which is one of the kidney functions. The GFR is typically recorded in units of volume per time, e.g., millilitres per minute $(\mathrm{ml} / \mathrm{min})$. Glomerular filtration rate (GFR) is the best overall index of kidney function, which is the best way to tell how well his kidney is working. It is a more sensitive method of renal impairment than serum creatinine. Even with normal range of serum creatinine, person can have significantly reduced renal function and by the time the serum creatinine is increased above normal range, there could be reduction in up to $50 \%$ of renal function. Earlier identification is possible in patients with chronic kidney disease (CKD) using GFR. This is 
particularly important as these patients are at increased cardiovascular risk compared with the general population and may benefit from risk factor modification. Normal GFR is above $100 \mathrm{ml} /$ minute $/ 1.73 \mathrm{~m}^{2}$. A GFR in the range of $60-100$ may indicate early renal disease. A GFR under 60 $\mathrm{ml} / \mathrm{minute} / 1.73 \mathrm{~m} 2$ indicates significant kidney disease. Lower GFR value indicates worse kidney function. Also, a progressive decline in GFR is an indicator of declining in the renal function. ${ }^{3}$ In clinical practice GFR is estimated (eGFR) using a mathematical formula that considers a person's body surface area, age, sex, race and serum creatinine levels. Presently eGFR is calculated using modified diet in renal disease (MDRD) formula which gives eGFR in $\mathrm{ml} /$ $\min / 1.73 \mathrm{~m}^{2}$. The MDRD formula is derived at $(175 \times$ $(\mathrm{Scr})-1.154 \times($ Age $)-0.203 \times(0.742$ if female $) \times(1.212$ if African American). The estimated GFR using this formula is found to be more accurate and acceptable in evaluating kidney disease in day to day practice. ${ }^{4-6}$ The objective of the present study was to compare the sonographic severity grading of renal parenchymal changes with eGFR calculated using MDRD formula among the patients with bilateral renal disease. Study aimed to evaluate the relevance of sonographic grading of renal parenchymal changes in assessing the severity of the renal disease and comparing it to the eGFR calculated using MDRD formula based on the age, gender and serum creatinine value of the patient.

\section{MATERIAL AND METHODS}

The study was conducted in the Department of Radiology, in collaboration with Department of Medicine and Nephrology, Bangalore Medical College Hospital, Bangalore, India.

Patients with suspected kidney disease referred to the department of radio-diagnosis, Bangalore medical college and research institute, for sonography of abdomen are the source of data.

A prospective descriptive study was conducted from November 2017 to May 2019 with a sample size of 80 cases. Age $>18$ years (MDRD formula for calculating eGFR is applicable for persons aged more than 18 only) and patients with bilateral disease are included in the study.

Those patients on renal replacement therapy like dialysis or renal patients with major physical handicap, patients with single kidney, obstructive uropathy, cases of polycystic kidney disease, renal malignancy, and patients in sepsis, having acute illness like diarrhea, dehydration and acute febrile illness are excluded from the study.

We did not include or exclude the patients for the study based on history of diabetes or hypertension. For each of the patient with suspected renal disease ultrasound scan of abdomen was performed with Phillips Affinity using convex transducer by a single radiologist and the renal size was measured, renal parenchymal status was documented based on their size and echo-texture.

The echo texture of spleen instead of liver is used to compare the renal cortical echotexture, as presence of fatty liver may cause errors in comparison and splenic echo texture is consistently preserved in majority of population.

Following grading system was used.

\section{Sonographic grading of renal parenchymal changes}

Grade 0 - Normal sized kidney, cortical echogenicity is less than that of spleen, with well-maintained cortico-medullary differentiation.

Grade 1 - Normal sized kidney, cortical echogenicity is same as that of spleen, with maintained cortico-medullary differentiation.

Grade 2 - Normal sized kidney, cortical echogenicity is more than that of spleen, decreased cortico-medullary differentiation.

Grade 3 - reduced renal length, cortical echogenicity is more than that of spleen, with poorly maintained corticomedullary differentiation

In clinical practice GFR is estimated (eGFR) using a mathematical formula that considers a person's body surface area, age, sex, race and serum creatinine levels. Presently eGFR is calculated using modified diet in renal disease (MDRD) formula which gives eGFR in $\mathrm{ml} / \mathrm{min} / 1.73 \mathrm{~m}^{2}$.

The MDRD formula is derived at $(175 \times(\mathrm{Scr})-1.154 \times($ Age $)$ $0.203 \times(0.742$ if female $) \times(1.212$ if African American $)$.

The equation does not require weight because the results are reported normalized to $1.73 \mathrm{~m}^{2}$ body surface area, which is an accepted average adult surface area.

Ethnicity of the person is considered in calculating eGFR using MDRD formula, because Africans tend to have proportionally greater muscle mass than non-Africans and therefore produce more creatinine.

\section{STATISTICAL ANALYSIS}

The data was analysed using Statistical Package for the social sciences (SPSS) for windows version 16.0.Chicago, SPSS Inc. Descriptive statistics were used to express the results. Pearson or Spearman's correlation coefficient values were used to draw valid inferences. All the patients of our study were of Indian origin, residents of Karnataka.

\section{RESULTS}

A total of 80 patients were our study participants, out of which 52 (67.1\%) were males and 28 (32.9\%) were females. The mean age of the study participants was $52.5+/-7.8$ years in males and 57.3/-9.3 in females (Table-1). There was no specific pattern of sonographic grading in different age groups. The findings were not gender dependent and the findings were the same in both sexes. On sonographic evaluation of our study participants; $15(20 \%)$ cases showed grade "0", $12(15.7 \%)$ cases showed grade " 1 ", 34(42.9\%) cases showed grade "2" (Figure-1) and19 (21.4\%) cases showed grade "3" renal parenchymal changes (Table-2). There was no change in grading of renal parenchymal changes between male and females or patients of different age groups. In our study, as the sonographic grading of renal parenchymal changes increased, the serum creatinine also increased in linear pattern. The tendency of the graph showed linear correlation between the sonographic grading of renal parenchymal changes and serum creatinine. A graph generated by plotting sonographic grading of renal parenchymal changes and eGFR. This graph shows an inverse relationship between the sonographic grading and eGFR. As the grading of 


\begin{tabular}{|l|c|c|}
\hline \multirow{2}{*}{ Parameter } & \multicolumn{2}{|c|}{ Gender } \\
\cline { 2 - 3 } & $\begin{array}{c}\text { Male } \\
(\mathbf{n}=\mathbf{5 2})\end{array}$ & $\begin{array}{c}\text { Female } \\
(\mathbf{n}=\mathbf{2 8})\end{array}$ \\
\hline Mean age(+/-SD) & $52.5+/-7.8$ & $57.3 /-9.3$ \\
\hline Mean kidney size (+/-SD) (cm) & $9.8+/-0.85$ & $9.3+/-1.15$ \\
\hline Serum creatinine (+/-SD) (mg/dl) & $2.1+/-0.93$ & $2.1+/-1.2$ \\
\hline eGFR (+/-SD) (ml/min/1.73m²) & $46.8+/-30.4$ & $38.4+/-25.5$ \\
\hline \multicolumn{2}{|c|}{ Table-1: Mean distribution of patients } \\
\hline \multicolumn{2}{|c|}{}
\end{tabular}

\begin{tabular}{|l|l|l|}
\hline \multicolumn{2}{|l|}{ Sonographic grading } \\
\hline Grades & Males & Females \\
\hline 0 & 9 & 6 \\
\hline I & 8 & 4 \\
\hline II & 24 & 10 \\
\hline III & 11 & 8 \\
\hline \multicolumn{2}{|l|}{ Table-2: Distribution of sonographic grading among patients } \\
\hline
\end{tabular}

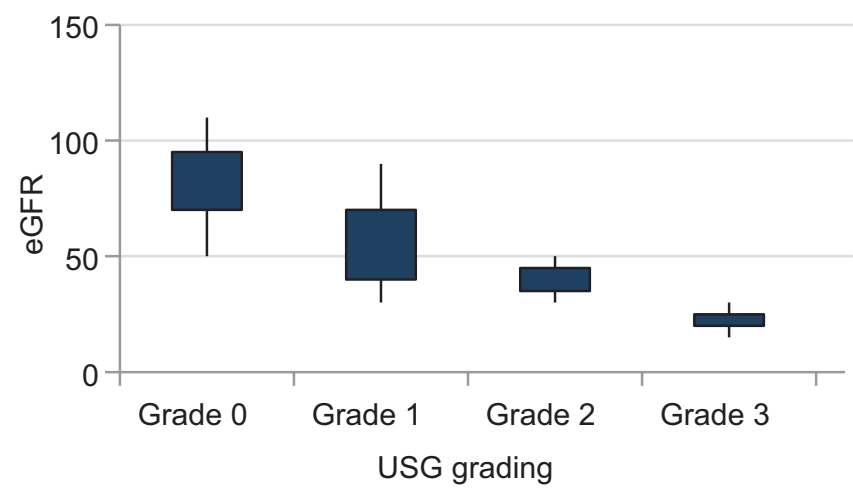

Graph-1. Distribution of relationship between USG grading of renal parenchyma and eGFR

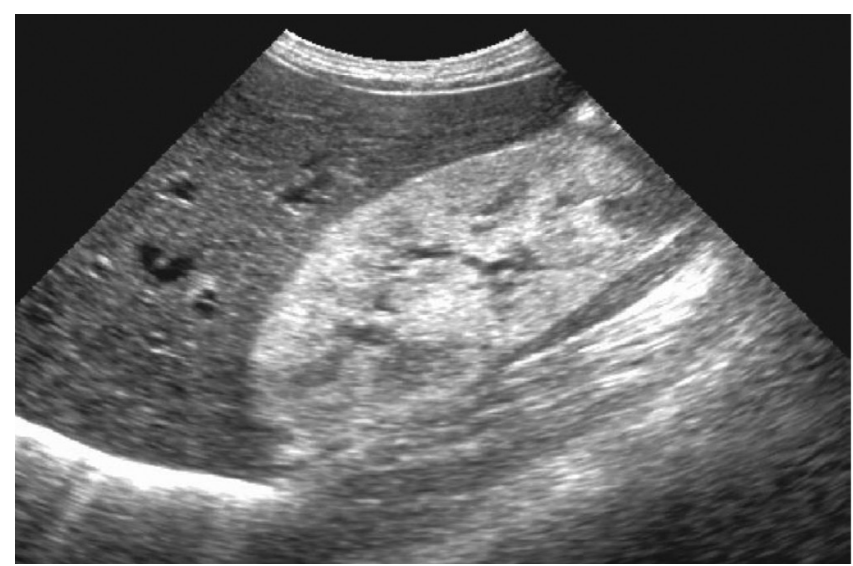

Figure-1: Normal sized kidney, cortical echogenicity is more than that of spleen, decreased cortico-medullary differentiation

renal parenchymal changes increased, the eGFR decreased (Graph-1). Similarly the serum creatinine had an inverse relationship with eGFR; i.e. as the serum creatinine increased, the eGFR decreased. The other finding we noted was a correlation between the kidney size and eGFR. As the average kidney size decreased, eGFR also decreased in linear pattern. Our study showed a linear correlation between sonographic grading of renal parenchymal changes with eGFR.

\section{DISCUSSION}

Sonography is an important non-invasive investigating tool in evaluation of diseases of the kidney according to study conducted by Kalantarinia $\mathrm{K}$ et al. ${ }^{7} \mathrm{By}$ the renal parenchymal changes grading, the severity of the disease can be assessed. ${ }^{8}$ GFR is the most important indicator of renal function. By calculating eGFR using simple available MDRD formula, the reserved renal function and in turn the severity and prognosis of the patient can be evaluated. ${ }^{9}$ Study conducted by Huntington DK et al., have shown accuracy of sonography in assessing the renal parenchymal disease with good sensitivity, though less specificity. ${ }^{10}$ Levey et al., have studied the role of eGFR in evaluating chronic kidney disease and in predicting prognosis. The eGFR is one of the important indicator of reserved renal function and the indicator of prognosis. Progressive declining eGFR is associated with higher morbidity and mortality. ${ }^{11}$ In our study we attempted in comparing the sonographic grading of renal parenchymal changes, which is useful in initial evaluation of renal disease with the eGFR which is an indicator of reserved renal function and prognosis. We used two independent diagnostic tools to enhance the accuracy of renal assessment. In our study, the correlation between the sonographic grading and the eGFR showed that, increased grading was associated with decreased eGFR. Hence, Sonography is the preliminary investigating tool, as it is easily available, non-invasive and does not involve exposure to ionizing radiation. By considering eGFR and sonographic grading of renal parenchymal changes the accuracy of assessment of renal ruction will be increased.

\section{Limitations of the present study}

Neither sonography nor eGFR can point out the specific etiology for compromised renal function.

\section{CONCLUSION}

EGFR is an important factor which can be used as a factor to predict prognosis in renal diseases. Sonography is a good non-invasive diagnostic tool for evaluation of renal disease. There is good correlation between the sonographic grading and eGFR using MDRD formula. We conclude that by evaluating the kidneys with Sonography and calculating eGFR using MDRD formula the renal status will be more accurately interpreted.

\section{REFERENCES}

1. O'Neill WC. Sonographic evaluation of renal failure. Am J Kidney Dis. 2000;35(6):1021-38.

2. Page JE. Morgan SH, Eastwood JB, Smith SA, Webb DJ, Dilly SA, et al. Ultrasound findings in renal parenchymal disease: comparison with histological appearances. Clin Radiol. 1994;49(12):867-70.

3. Jerums G, Ekinci E, Panagiotopoulos S, MacIsaac RJ. Early Glomerular Filtration Rate Loss as a Marker of Diabetic Nephropathy. European Endocrinology. 2012;8(1):27-31.

4. Levey AS. Bosch JP, Lewis JB, Greene T, Rogers N, Roth D. A more accurate method to estimate glomerular filtration rate from serum creatinine: a new prediction equation. Modification of Diet in Renal Disease Study Group. An Intern Med. 1999;130(6):461-70. 
5. Siddappa JK, Singla S, Al Ameen M, Rakshith SC, Kumar N. Correlation of Ultrasonographic Parameters with Serum Creatinine in Chronic Kidney Disease. J Clin Imaging Sci. 2013;3(4):28.

6. Brandt TD, Neiman HL, Dragowski MJ, Bulawa W, Claykamp G. Ultrasound assessment of normal renal dimensions, J Ultrasound Med. 1982;1(2):49-52.

7. Kalantarinia K. Novel imaging techniques in acute kidney injury. Curr Drug Targets. 2009;10(12):1184-89.

8. Page JE, Morgan SH, Eastwood JB, Smith SA, Webb DJ, Dilly SA, et al. Ultrasound findings in renal parenchymal disease: comparison with histological appearances. Clin Radiol. 1994;49(12):867-70.

9. National Kidney Foundation. Bailie GR, Uhlig K, Levey AS. Clinical practice guidelines in nephrology. Evaluation, classification, and stratification of chronic kidney disease. Am J Kidney Dis. 2002;39(suppl 2):S1266.

10. Huntington DK, Hill SC, Hill MC. Sonographic manifestations of medical renal disease. Semin Ultrasound CT MR. 1991;12(5):290-307.

11. Levey AS, Coresh J, Balk E, Kausz AT, Levin A, Steffes MW, et al. National Kidney Foundation practice guidelines for chronic kidney disease: evaluation, classification, and stratification. Ann Intern Med. 2003;139(2):1.

Source of Support: Nil; Conflict of Interest: None

Submitted: 22-02-2020; Accepted: 13-04-2020; Published online: 10-05-2020 